\title{
Beitrag zur Kennntniss der normalen Bindehaut des Menschen.
}

Von

Professor H. Sattler.

$\mathrm{D}_{\mathrm{a}}$ ich über einige seltene und eigenthümliche Bindehaut-Erkrankungen zu berichten haben werde, so stellte sich das Bedürfniss heraus, den normalen Zustand der Bindehaut vorher noch einer genauen Prüfung zu unterziehen, da trotz der eingehenden und verdienstvollen Arbeiten der neuesten Zeit über diesen Gegenstand in Betreff mehrerer Punkte eine Uebereinstimmung noch nicht hat erzielt werden können, und auch meine eigenen, schon früher darüber gewonnenen Erfahrungen in einigen Punkten differirten. Als die streitigsten und unsichersten Gebiete in der Anatomie der Conjunctiva bezeichnet $\mathrm{Reich}$ *) sehr richtig: die sogenannten blinddarmförmigen Drüsen von Henle, beziehnngsweise den sogenannten Papillarkörper, ferner das Epithel der Conjunctiva mit Einschluss der sog. Becherzellen, und endlich das adenoide Gewebe derselben.

*) Zur Histologie der Conjunctiva des Menschen. Archiv für Ophthalm. XXI, 1, pag. 2. 1875.

v. Graete's Archiv fux Ophthalmologie, XXIII. 4 . 
Wenn man eine recht grosse Anzahl von Bindehäuten, die man bei der Betrachtung mit blossem Auge als normal bezeichnen zu dürfen glaubt, einer genauen Untersuchung in ihrer ganzen Ausdehnung unterwirft, so überzeugt man sich bald, dass gerade in Bezug auf die oben angedeuteten' Punkte gar nicht unerhebliche Differenzen bestehen, sowohl zwischen verschiedenen Bindehäuten von Erwachsenen, als namentlich zwischen den von Erwachsenen und Kindern verschiedenen Alters. Dazu kommt noch, dass Schnitte aus einer und derselben Lidbindehaut ein wechselndes Aussehen darbieten, je nachdem sie aus der Mitte oder näher einem der beiden Winkel genommen sind. In den vorliegenden Darstellungen des histologischen Baues der Conjunctiva finden wir gewöhnlich ein bestimmtes Schema entworfen, welches mehr oder weniger vollständig auf eine gewisse Summe von Bindehäuten passt, bei anderen aber nicht zutrifft.

Schon bei Betrachtung der Tarsalbindehaut mit der Loupe müssen die Differenzen auffallen, welche in Bezug auf die Entwickelung und Ausdehnung der sammetartigen Beschaffenheit ihrer Oberfläche bei verschiedenen Individuen obwalten. Dass dieses sammetartige Aussehen von der Anwesenheit zahlreicher zarter Erhabenheiten der Schleimhaut-Oberfläche herrühre, ist zwar seit langer Zeit bekannt; doch wurden die letzteren lange als Drüsen oder Drüsen-Ausführungsgänge gedeutet, und erst seit Eble's, seiner Zeit epochemachender Bearbeitung dieses Gegenstandes*) ziemlich allgemein als Papillarkörper bezeichnet, sowohl von den Anatomen, als in den zahlreichen Handbüchern der Augenheilkunde. Erst in den Darstellungen der nevesten Zeit ist von

*) Ueber den Bau und die Krankheiten der Binnehaut u. s. W. Wien 1828. 
einem Papillarkörper nicht mohr die Rede (Henle, Stieda, Merkel, Waldeyer, $\left.{ }^{*}\right)$ ) oder er wird geradezu geleugnet (Wolfring, Ciaccio, Morano,**)) und man spricht nur mehr von rinnenartigen Vertiefungen, Furchen und Grübchen, welche die Conjunctiva tarsi nach allen Richtungen durchsetzen.

Erst Reich (1. c., pag. 13) nimmt sich der Papillen wieder an, und behauptet, dass "ausser denjenigen Erhabenheiten und Falten, die durch unregelmässige Furchen und Einschnitte gebildet werden, . . . . . auch wirkliche Papillen zu finden sind."

Man muss allerdings gestehen, dass diese Gebilde etwas anders gebaut sind, als die bekannten conischen oder cylindrischen Papillen der Haut und vieler Schleimhäute, so der Schleimhaut der Mundhöhle, des Oesophagus, der Vagina und anderer. Solche im strengen Sinne der Anatomen wahre Papillen finden sich an der Lidbindebaut nur an der inneren Lidkante, worauf auch Wolfring (1. c., p. 161) und Ciaccio (1. c., p. 9) aufmerksam machen. Hier füllt das mehrfach geschichtete Epithel die Einsenkungen zwischen den Papillen vollkommen aus, so dass dieser Streifen oberflächlich glatt erscheint, ***) und selbst dann ziemlich glatt bleibt, wenn in pathologischen Processen die übrige Lidbindehaut ganz uneben und warzig geworden ist; auch besitzen

*) Henle, Eingeweidelehre, pag. 702.

Stieda, Arch. f. mikrosk. Anat., III, pag. 357.

Merkel, Handbuch d. ges. Augenheilk., I. Bd., I. Th, p. 67. Waldeyer, ibidem, pag. 240.

**) Wolfring, Arch. f. Ophth, XIV, 3, pag. 160.

Ciaccio, Osservazioni intorno alla Struttura della Congiuntiva umana. Bologna 1874.

Morano, Sezione meridionale della Conginntiva umana. Annali di Ottalmol. Anno VI., Fasc. I., pag. 21.

***) Vergl. die treffliche Abbildung Walde yer's (Fig. 26) im Handbuch der ges. Augenheilkunde $v$. Graefe . Saemisch, I. 
wenigstens die grösseren dieser Papillen eine eigene kleine Arterie, welche sich darin in schlingenförmig angeordnete Capillaren auflöst. An der Tarsalbindehant hingegen macht das Epithel alle stärkeren Niveandifferenzen der Tunica propria mit - dadurch gewinnt eben dieser Abschnitt der Conjunctiva sein sammetartiges Aussehen -; und auch die Blntcapillaren, welche gleich unter der freien oberfläche ausgebreitet sind, zeigen eine ziemlich gleichmässige netzfôrmige Anordnung in der ganzen Ausdehnung der Tarsalbindehaut, in den Papillen so gut, wie in der Tiefe der Rinnen. Da dieses Capillarnetz von zahlreichen kleinen Arterien gespeist wird, welche von den der hinteren Fläche des Tarsus entlang laufenden gröberen Verzweigungen ihren Ursprung nehmen, so ist es selbstverständlich, dass wir in viele der kleineren und sämmtliche grösseren Erhebungen arterielle $Z$ weigchen aufsteigen sehen.

Wie immer man nun vom anatomischen Standpunkte diese Gebilde auffassen mag, für den Kliniker ist es jedenfalls bequem, von Papillen und einem Papillarkörper sprechen zu können, wenn nur einmal der Begriff, der hier damit verbunden werden soll, bestimmt festgestellt ist.

Die Ausbreitung und Entwickelung dieses Papillarkörpers variirt nun bei verschiedenen Individuen ungemein. An einer Reihe von Tarsalbindebäuten Erwachsener*) erscheint die Oberfläche, für's freie Auge wenigstens, in grosser Ausdehnung vollkommen glatt und eben, und erst in der Nähe des

*) Der Bindehaut von Nengeborenen und spateren Embryonen werden wix im Folgenden noch mit einigen Worten zu gedenken haben. Ich bemerke nur hier gleich, dass die oben citirte, treffliche Abbildung Waldeyer's, nach meinen Präparaten zu urtheilen, in Bezug auf die Entwickelung der Papillen einem neugeborenen oder wenige Tage alten Kinde entnommen sein muss, 
convexen Randes, ungefähr im hinteren Drittel, so wie gegen die beiden Winkel zu sieht man rundliche Hügel, welche allmälig an Grösse zunehmen, mit ihrer leicht abgeplatteten Oberfläche sämmtlich in einem Niveau liegen, und gleich jenseits des convexen Randes wieder schwinden. In vielen anderen Fällen wieder beginnen die Erhebungen als kleine, mit freiem Auge eben wahrnehmbare Hügelchen schon $1^{1} / 2-2 \mathrm{Mm}$. vom freien Rande, gewinnen gegen den convexen Rand und die beiden Winkel zu mehr oder weniger rasch an Höhe und Mächtiglseit, und erstrecken sich auch noch uber denselben hinaus auf die erste bis zweite Querfalte der Pars orbitalis. In solchen Fällen sind die Erhebungen am convexen Rande und jenseits desselben auch nicht mehr sämmtlich in gleichem Niveau, sondern cinzelne Papillen oder Gruppen von solchen ragen als flache Inselu da und dort über die übrigen etwas cmpor. Diesem letztgeschilderten Typus der Conjunctiva tarsalis und orbitalis begegnet man sohäufig, und er stimmt auch mit dem von einigen Autoren gegebenen Schema ihres Baues so nahe überein, dass man ihn noch kaum als pathologisch bezeichnen dürfte. Sicher bildet er aber die äusserste Grenzmarke des normalen. Zwischen den beiden im Vorhergehenden gezeichneten Extremen findet man nun die mannigfaltigsten Abstufungen.

Was die viel umstrittenen Einsenkungen und "tubulösen Drüsen" betrifft, so muss ich gestehen, dass ich bei der grossen Anzahl von Lidern, die ich untersuchte, entweder ausschliesslich oder wenigstens in der weitaus überwiegendsten Ausdehnung blos Rinnen, Furchen, nach allen Richtungen verzweigte und nnter einander zusammenhängende Thäler angetrofien habe, welche, nach der beite des Lidrandes zu immer seichter und enger werdend, ganz allmälig auslaufen, und nach dem Orbithaltheil hin ohne Grenze in 
die hier befindichen Querfalten übergehen; und dass nur verhältnissmässig spärlich is olirte kurze Rinnen, die einfach oder schwach verzweigt sein können, oder grübchenartige Vertiefungen zu finden waren, welche in grössere, unregelmässig gestaltete Erhebungen eingegraben erschienen. Beachtenswerth für das Verständniss der divergenten Darstellungen, welche diese Gebilde erfahren haben, ist noch der Umstand, dass der Grund der Furchen bei weitem nicht uberall in gleicher Tiefe liegt, sondern bald seichter, bald tiefer ist, und da und dort wohl auch kurze blindendigende Ausstülpungen zeigt. (Vergl. auch Stieda, 1.c., pag. 362.)

Von der Richtigkeit der gegebenen Darstellung bezüglich des Verhältnisses der Erhebungen zu den Einsenkungen kann man sich schon bei aufmerksamer Betrachtung der BindehantOberfläche mit einer guten Lonpe uberzeugen. ${ }^{*}$ ) Noch zwingender ist der Eindruck, den man durch richtig ausgeführte Flächenschnitte erhält, wie sie Stieda und spätere Autoren ausfuhrten. Dicke und hinreichend aufgehellte Schnitte geben bei wechselnder Einstellung das schonste Relief in dem oben angedeuteten Sinne; und feine Schnitte liefern verschiedene Bilder, je nachdem sie näher der Oberfläehe oder der Basis des Papillarkörpers entnommen sind. Im orsteren Falle bekommt man, wenn man dafür sorgt, dass die Finbettungsmasse entweder mit eingeschlossen wird, oder erst auf dem objectiräger sich löst, durchaus rundliche, ovale oder auch unregelmässig gestaltete isolirte Inseln von verschiedener Grösse, welche allseitig von hellen, theils engeren, theils weiteren Räumen umflossen sind, und nur da und dort durch eine schmalere oder breitere Brücke, dio erst bei geänderter Einstellung deutlich wird, verbunden erscheinen. Nur ausnahmsweise findet sich innerhalb einer der grösseren Inseln ein runder epithelbekleideter Durchschnitt einer grübchenartigen

*) Wenn es bei einem Object, das man sich jederzeit so leicht verschaffen kann, noch nöthig wäre auf eine Abbildung hinzuweisen, so könnte Fig. 70 in $\mathrm{Th}$. Nunnele $\mathrm{y}^{3} \mathrm{~s}$ On the organs of vision ete, London 1858 , genannt werder. 
Einsenkung. Andere, als die eben beschriebenen Bilder bekommt man an Schnitten dieser Art ron der Tarsalbindehaut nicht zu sehen.*) Anders ist es, wenn Schnitte von der Nahe der Basis des Papillarkörpers durchmustert werden. Hier erscheinen dio Parenchyn-Inseln ron viel unregelmässigerer Form, hängen häufig unter einander zusammen, und man trifft viele unterbrochene einfache oder verzweigte, sowie auch einzelne rundliche Durchschnitte von scheinbaren Kanälen all dies der Ausdruck der Tiefendifferenzen am Grunde der oben beschriebenen Einsenkungen.

Ich habe mich bei diesem Gegenstande länger aufgehalten, um nach zahlreichen Untersuchungen ein möglichst getreues Bild der scheinbar so bekannten Verhältnisse zu entwerfen, damit der Leser leichter selbst beurtheilen könne, ob die Existenz einfacher und zusammengesetzter tubulöser Drüsen, welche in neuesten Arbeiten wieder auf's Angelegentlichste vertheidigt wird, eine Berechtigung habe. Nachdem Ciaccio, 1. c, p. 17) beschrieben hat, dass einige dieser "Henle'schen Schleimdrüsen" wie Bläschen oder Säckchen aussehen, andere die Form eines verschieden langen, geraden oder gekrümmten oder leicht gewundenen Canals oder Schlauches haben, welcher sich, wenn auch selten, theilen kann (Glandule tubulose composte), so spricht er noch von einer dritten Art von Drüsen, welche nichts Anderes seien, als blosse Aushöhlungen (Scanalature) in der Mucosa, bald tiefer, bald weniger tief, und meistentheils unter einander zusammenhängend. Diese Aushöhlungen würden in vollständige Kanäle umgewandelt durch das Epithel der Bindehaut, welches darüberziehe und sie schliesse, und nur da und dort einige kleine Mündungen

*) Wenn man in der Fig. 3 von Ciaceio die Verbältnisse gerade umkehrte, d. h. die "Glandule mucose" als die ParenchymInseln betrachtete, so entspräche die Figur viel besser den gewöhnlichen Verhältnissen. 
offen lasse, durch welche der Schleim austreten lkönne, welcher beständig in ibnen secernirt werde (1. c., pag. 13 und 17). Seine einfachen, bläschen- oder säckchenförmigen Schleimdrüsen entsprechen offenbar den überhanpt nur spärlichen, bisweilen auch ganz fehlenden grübchenartigen Einsenkungen (siehe oben). Die Form von Säckchen oder Bläschen nehmen sie nur an durch pathologische Umwandlung des Epithels im Grunde der Einsenkung in sogenannten Becherzellen, wobei der Grund erweitert wird, während die Oeffnung durch gleichzeitige geringe Schwellung der Schleimhaut-Erhebung, in welcher sie sich befindet, eng bleibt. Was nun aber die zweite und dritte Art von Drüsen. die Kanäle und Schläuche anlangt, so lagen hier entweder ebenfalls pathologische Zustände oder ungenügende Präparate vor. Selbst zugageben, dass es sieh so verhielte, wie Ciaccio angiebt, dass nämlich die Aushöhlungen durch das Epithel bis auf kleine Oeffnungen geschlossen würden, so würde uns dies nach dem uns geläufigen Begriff ron Drüsen noch nicht berechtigen, diese Gebilde als Drüsen $z u$ bezeichnen. Uebrigens lehrt ein Blick auf Fig. 1, welche der Fig. 545 in Henle's Eingeweidelehre (pag. 702) volikommen analog ist, dass das Epithel nicht mehr intact war, und vielleicht abgefallene oder zertrümmerte Epithelzellen die Eingänge zu den Einsenkungen verlegten. Weder auf der Höhe der Papillen (als Tramezzi und Ajuole bezeichnet), noch in den rimnenartigen Vertiefungen entspricht das Epithel in der Abbildung den factischen Verhältnissen; an beiden Localitäten ist es nämlich, wie Waldeyer und Reich als die einzigen unter den zahlreichen Autoren richtig beschreiben und abbilden, und Wolfring noch etwas unbestimmt ausspricht, ein schön entwickeltes Cylinder-Epithel, das aus einer unteren Lage kleiner polyedrischer oder rundlicher Zellen von 0,006-0,008 Mm. 
Höhe, und einer oberen Lage mehr oder weniger hoher und schlanker Cylinderzellen besteht. So lange die Einsenkungen noch wenig tief sind, erscheinen die Cylinderzellen sämmtlich von ziemlich gleicher Höhe; mehr gegen den convexen Rand zu sind sie auf der Höhe der Papillen ausgesprochen conisch, mit deutlichen Fortsätzen am Basal-Fnde (siehe Reich, 1. c., pag. 6), und von ca. 0,016-0,020 Mm. Höhe; in den Buchten dagegen schlanker, häufig von mehr unregelmässiger Form und in der Regel entschieden höher, bis 0,025$0,030 \mathrm{Mm}$. Aber diese Anordnung findet sich an Präparaten, wo das Epithel tadellos erhalten und nicht durch pathologische Vorgänge, die hier allerdings nicht selten angetroffen werden, verändert ist, gleichmässig im ganzen System der Einsenkungen, nicht blos in den "Ausführungsgängen tubulöser Drüsen" (Reich, pag. 7 und 14). Damit ist auch im Einklang mit Wolfring (1. c., pag. 163), Waldeyer, Ciaccio (1. c., pag. 19) und Reich (l. c., pag. 16) der Ansicht derjenigen widersprochen, welche wie Henle (l. c., Fig. 545), Stieda (l. c., Taf. XX, Fig. 4) und Zehender (Handbuch der ges. Augenheilk., I. B., 1874, pag. 52) "die blinddarmförmigen Drüsen", resp. „die Furchen und Einschnitte" von einer einfachen Lage hoher Cylinderzellen aus. gekleidet werden lassen, etwa wie die Lieberkühn'schen Krypten des Darmkanals. Ich glaube darauf besonderes Gewicht legen zu müssen, dass kein irgend wesentlicher Unterschied zwischen dem Epithel der Papillen und dem der Einsenkungen besteht - wir werden später noch einmal darauf zurüekkommen und es scheint mir selbst die Ansicht, die Waldeyer hinstellt, dass die furchenartigen Einschnitte als schleimabsondernde drüsige Bildungen fungiren könnten (1. c., pag. 240), für den normalen Zustand der Bindehaut wenigstens, unannehmbạ zu sein, weil ich nach klinischen 
Erfahrungen bestreiten muss, dass die normale Bindehaut überhaupt ein sehleimiges Secret liefere, wie Ciaccio als ausgemacht anzunehmen scheint (1. c., pag. 18 oben). Das Secret der Bindehaut ist ein wässeriges, dem der Thränendrüse analoges, vielleicht auch vollkommen gleiches (vergl. Arlt, die Krankh. des Anges, I, pag. 6, und de Wecker, Traité théor. et pract. des maladies des yeux, I, 2me. Edit., pag. 9), wie man sich jeder Zeit am Lebenden überzeugen kann, wenn man das obere Lid umstulpt und sorgfältig abtrocknet. In wenig Angenblicken ist es wieder feucht, und nicht etwa vom convexen Rand her, in dessen Nähe die Ausführungsgänge der Thränendrüsen und der acinotubulären Drüsen (Waldeyer) liegen, sondern gleichzeitig an der ganzen Oberfäche.

Wenn schleimige Umwandlung der Epithelzellen stattgefunden hat, welche im Bereiche des CylinderEpithels immer die obere Zellsehicht betrifft, so fand ich allerdings diese "Schleim-" oder Becherzellen" viel zahlreicher, bisweilen ansschliesslich in den Buchten, während die Oberfläche der Erhebungen intacte Cylinderzellen trug. Aber einerseits muss ich diese Veränderung, welche von Ciaccio (l. c., pag. 15 und 19) und Reich, (1. c., pag. 9) mit grosser Ausführlichkeit beschrieben worden ist, im Einklang mit den genannten Autoren für entschieden pathologisch erklären,*) andererseits

*) Es sind mehr oder weniger leichte catarrhalische Reizzustände der Bindehaut, Fälle von chronischen Granulationen, chronischer Blennorrhoe und Trachom, wobei die in Rede stehende Unwandlung cylindrischer Epithelzellen zur Beobachtung kommt. Anch habe ich dieselbe ebenso, wie Ciaccio und $R$ eich verhaltnissmässig hänfig beỉ alten Individuen gesehen, die bekanntlich recht oft an leichtem chronischen Conjunctivalcatarrh leiden; dann aber auch einmal bei einem wenige Wochen alten Kinde. Ich vermisste sie vollständig bei acuter Bindehautblennorrhoe (Blennorrhoea neonatorum) und bei den sog. acuten Granulationen. 
kann ich versichern, dass ich in einigen Fällen auch in der Pars orbitalis, jenseits der Grenze des Papillarkörpers, in den Furchen zwischen den horizontalen Falten sämmtliche Cylinderzellen in schleimiger Umwandlung antraf, während auf der Höbe der Falten die Cylinderzellen unverändert blieben. Demnach könnte man nicht einmal unter diesen pathologischen Verhältnissen die furchenartigen Einsenkungen zwischen den Papillen als die wesentlich schieimabsondernden Organe ansehen, sondern es scheinen eben nur die "Schleimzellen" in den Yertiefungen länger zurückgehalten zu werden, während sie an der Oberfläche rasch abgestossen und durch nene intacte Zellen wieder ersetzt werden. Durch diese schleimige Umwandlung des Epithels können die Rinnen und Buchten an umschriebenen Stellen mehr oder weniger beträchtlich erweitert werden, und wenn nun bei gleichzeitiger, wenn auch nur mässiger Anschwellung der Tunica propria conjunctivae da und dort die Eingänge zu den Rinnen sich abschliessen, so hat man kleine mikroskopische Cysten (Tumoretti cistici Ciaccio) vor sich, in denen man nicht selten schleimige Massen angesammelt findet, und welche sich am Lebenden als kleinste durchscheinende Bläschen präsentiren.

Um das Platten-Epithel auf der Oberfläche der Erhebungen zu retten, nehmen einige Autoren (Stieda, 1. c., pag. 363, Zehender, l. c., pag. 52) an, dass sich im späteren Leben, „,und zwar offenbar in Folge des beständigen Druckes, welcher zwischen dem Augapfel und den Augenlidern stattfindet," das Cylinder-Epithel auf der Oberfläche, welches sie für das Kindesalter zugeben, in ein geschichtetes Platten-Epithel umwandle. So sehr diese Lösung anf den ersten Blick annehmbar scheint, so ist sie gleichwohl unrichtig; und es scheint diese Annahme wohl nur darin ihren Grund zu haben, dass man sich viel leichter frische Kinderleichen versehaffen kann, als solche von Erwachsenen. Wenn man in der Lage ist, auch von Erwachsenen verschiedener Lebensperioden gut erhaltene Präparate zu bekommen, so kann man sich mit Leichtigkeit 
von dem constanten Vorkommen des Cylinder-Fipithels anf der: Oberfläche der Erhebungen überzengen. Dass die Cylinderzellen hier in der Regel weniger hoch sind als in den Einsenkungen, wurde oben zugegeben, aber zugleich betont, dass sie ihre conische Gestalt nie dabei verlieren. Was ubrigens sehon von vornherein bei der Annahme eines solchen ans kleinen rundlichen und flachen Zellen bestehenden geschichteten Epithels, wie es von den genannten Autoren beschrieben und abgebildet wird, zur Vorsicht mahnen sollte, ist der Umstand, dass eine derartige Epithelform an keiner SchleimhautOberfläche des menschlichen Körpers wiederkehrt. Ja, noch mehr; wenn es in der That bei länger dauernden trachomatösen oder chroniseh-blennorrhoisehen Bindehant-Entzündungen zu einer Umwandluug des Cylinder-Epithels in Pflaster-Epithel gekommen ist, so finden wir das letztere auf der obertäche der Papillen und Körner gerade so gebaut, wie wir es an anderen Stellen zu sehen gewohnt sind, $d$. h. eine Lage cylindrischer Zellen unten, dann rundliche oder polygonale Elemente, und zuletzt mehrere Schichten mehr oder weniger abgeplatteter Zellen. Man hat da vielfach Gelegenheit, an der Mündung der rimnenartigen Einsenkungen den Vebergang der beiden EpithelArten zu studiren.*)

Das oben Angeführte gilt ebenso auch gegenïber der früher citirten Darstellung Morano's. Nicht viel besser begründet, als Ciaccio's Annahme von der Existenz tubulöser Drüsen ist die von Reich; letzterer stützt sich dabei auf Präparate (s. Fig. 5, 6 und 7), denen Beweiskraft nicht zugestanden worden kann. Unter den sehr zahlreichen Präparaten normaler Lider, die mir vorlagen, sind mir, sofern die Schnitte in ihrer ganzen Länge streng senkrecht waren, solche Bilder, wie

*) An einer anderen Localität, der hinteren, resp. oberen Fläche des Gaumensegels und der Uvala, scheint eine solche Umwandlung rom flimmernden Cylinder-Epithel des Neugeborenen zum gewöhnlichen geschichteten Pflaster-Epithel des Erwachsenen zur Regel zu gehoren, wie E. Kle in angiebt (Sitznngsbericht der k. k. Academie der Wissensck. zu Wien, 1868, Januar), und wovon ich mich selbst $\$$. Z. uberzeugte. 
die eben angedeuteten Figuren, nienals vorgekommen, weder jene verzweigten, blindendigenden Gänge, noch jene Querschnitte von Kanälen, noch auch jene ganz und gar eigenthümliche Gestalt der freien Oberfläche der Tunica propria. Alles dieses trifft man aber in der That, sobald man zufällig oder absichtlich vom oberen Theil der Tarsalbindehaut und der Pars orbitalis Schrägschnitte anfertigt, namentlich wenn der Papillarkörper etwas stärker entwickelt ist, und die Erhebungen in der Gegend des convexen Randes z. Th. keulenförmig, somit, die Eingänge zu den Rinnen enger sind, als der Grund derselben. Dies scheint bei den Bindehäuten, von denen Reich's Abbildungen entnommen sind, wirklich der Fall gewesen $\mathrm{zu}$ sein, wie aus der verhältnissmässig starken Entwickelung der Papillen und der lymphoiden Einlagerung in Fig. 1, welche aus der Mitte der Tarsushöhe stammt, zu ersehen ist. Je mehr die Schnittrichtung im Bereiche der Conjunctiva von der senkrechten sich entfernt, um so sicherer erhält man Bilder, die den ron Reich gegebenen Figuren gleichkommen (vergl. pag. 7 oben, die Beschreibung von Flächenschnitten). Wie leicht man gerade von dem obersten Theile der Conjunctiva tarsalis und von der Pars orbitalis Schrägschnitte bekommt, habe ich ans eigenen und meiner Schüler Arbeiten zur Genüge erfahren. Da das Gewebe hier lockerer und nachgiebiger ist, so weicht es leicht heim Schneiden etwas aus, oder ändert auch schon beim Einbetten seine Richtung.

Bezüglich des Epithels habe ich der Vollständigkeit halber noch einige Worte über den Uebergang des geschichteten Pflaster-Epithels der inneren Lidkante in das Cylinder-Epithel der Conjunctiva tarsi hinzuzufügen. Es existirt daruber nur eine kurze Bemerkung bei Waldeyer (l. c., pag. 239), und wir vermissen ganz und gar Angaben über den Ort des Uebergangs. Beim Erwachsenen traf ich im Durehschnitt schon $0,5 \mathrm{Mm}$. jenseits der inneren Lidkante schön ausgebildetes 
Oylinder-Eipithel; hier ist es aber noch staris gesehichtet, etwa wie das Epithel der Trachea, wenn man sich die Flimmerbärchen des letzteren wegdenlkt (siehe Waldeger, 1. c., Fig. 26). Bei einer Mächtigheit der ganzen Epitheldecke von 0,10-0,12 Mm, besizzen die obersten Cylinderzellon eine Höhe yon $0,012-0,016 \mathrm{Mm}$. Bei den Basalzellen überwiegt hier noch der Eöhendurchmesser mehr oder weniger den queren. Die Zellen der mittleren Lagen sind nicht selten nach beiden Seiten hin in Spitzen ausgezogen, mit denen sie sich zwischen die Nachbarn nach oben und unten einschmiegen. Circa $0,4 \mathrm{Mm}$. weiter anfwärts (oberes Lid), also ca. $0,9 \mathrm{Mm}$. über der Lidkante, ist das Epithel bereits auf 3 Schichten reducirt, ist im Ganzen nur mehr 0,04 MIm. dick; und bald darauf wird es zweischichtig; dabei werden die Basalzellen rasch niedriger, während die Cylinderzellen allmälig noch an Höhe zunehmen, bis 0,018 und $0,020 \mathrm{Mm}$. Beim Neugeborenen sind die Verhältnisse bezüglich des Epithels schon nahezu vollständig dieselben, wie beim Erwachsenen; nur ist das geschichtete CylinderEpithel nächst dem Lidrande etwas weniger dick, und reicht die Grenze zwischen Pflaster- und Cylinder-Epithel nicht etwa entsprechend der geringeren Höhe des Tarsus weniger weit hinauf, sondern befindet sich schon in derselben Hühe, d. $i$. ca. 0,5 Mrn. über der Lidkante. Bei mensehlichen Embryonen aus dem 6 . Schwangerschaftsmonate fand ich die besagte Grenze ziemlich constant sogar noch höher hinaufreichend (auf $0,7-0,8 \mathrm{Mm}$.). Obwohl schon etwas vor derselben die oberflächlichen Zellen weniger platst werden, so beginnen sie doch erst von dort an durch ihre ausgesprochen conische Gestalt sich als "Cylinderzellen" zu documentiren; vereinzelte Cylinderzellen oder ganz kleine Inseln von solchen finden sich zuweilen schon etwas früher zwischen den flachen Oberfiächenzellen. Da wo das Cylinder-Epithel seinen Anfang nimmt, beträgt die Mächtigkeit der Epitheldecke nur 0,012 $\mathrm{Mm}$. Die Gylinderzellen nehmen von da nur sehr allmälig an Höhe $\mathrm{zu}$, orreichen aber doch gegen den convexen Rand des Tarsus zut schon die Länge von $0,02 \mathrm{Mm}$. Hier zeichnen sie sich dann durch grosse Regelmässigkeit und Constanz in Form und Höbe aus.

In gleicher Weise schwankend, wie die Entwickelung des Papillarkörpers der Bindehaut ist die Aus- 
breitung und Ausbildung der lymphoiden Infiltration, und in gleicher Weise macht sich hier der Mangel an Uebereinstimmung zwischen den verschiedenen Autoren fühlbar. Während einige überhaupt über die Ausbreitung derselben kein Wort verlieren, und nur ganz allgemein ihre Existenz erwähnen (Kölliker, Stieda, Zehender u. A.), lassen sie Andere über den ganzen Tarsal- und Uebergangstheil (Ciaccio, 1. c., pag. 7, Reich, l. c., pag 18*), Morano, l. c., pag. 22), ja Henle sogar auf die conj. bulbi (1. c, pag. 704) sich erstrecken; und Waldeyer findet eine reticulirte Beschaffenheit des Stromas, ein adenoides Gewebe, bloss, in dem hinteren, dem Furchennetze der Bindehaut entsprechenden Abschnitte" entwickelt (l. c., pag. 240).

Man kann mit voller Präcision sagen, dass die Entwickelung und Ausbreitung der lymphoiden Infiltration in einem geraden Verhältniss steht zur Entwickelung und Ausbreitung des Papillarkörpers. (Vgl. oben p. 4 u. 5.)

Wenn wir zunächst die Bindehaut von ca. sechsmonatlichen menschlichen Embryonen in Augenschein nehmen, so finden wir die Oberfläche der ganzen Tarsalbindehaut (welche, am oberen Lid in dessen Mittellinie gemessen, eine Höhe von $3,12-3,15 \mathrm{Mm}$. hat) noch vollkommen glatt und eben. Thre Mächtigkeit nimmt vom freien Rand gegen den convexen allmälig $\mathrm{zu}$; (im Bereich des Pflaster-Epithels 0,02 $\mathrm{Mm}$. dick, hat sie beim Uebergang des letzteren in's Cylinder-Epithel (s. oben) bereits eine Mächtigkeit von $0,03-0,04$, und am oberen Ende des Tarsus von 0,05-0,06 Mm.). Sie wird gebildet aus einem Mattenwerk zarter Bindegewebsbündel, welche

*) Reich sagt wörtlich: Das Grundgewebe der Papillen und der obersten Schichten der Tunica propria conjunctiva uberhaupt ist sowohl auf dem Tarsaltheil als auch aufdem Fornix. theil (hier nur in den oberflächlichsten Schichten) ein reticuläres Stroma mit eingestreuten lymphoiden Zellen (adenoides Gewebe). 
vonugsweise in einer mit der Obertäche parallelen Ebene sich durchflechten, aber auch won zahlreichea Bündeln gekreuzt werden, welche von dem dichten Fasergeflecht des Tarsus entspringend in senkrechter oder mehr schräger Richtung der freien Oberfläche zustreben und dort pinselförmig ausstrahlen. (Wolfring, l. c., pag. 165, erwäbnt dieser Bündel zuerst). Gegen den freien Rand $z u$ ist dieses Mattenwerk dicht und straff; je mehr gegen den convexen Rand $\mathrm{zu}$, um so lockerer wird es, um so inehr treten die Bindegewebsbündel aus der mit der freien Ohertäche parallelen Ebene heraus, um sich in den verschiedensten Richtungen zu durchsetzen, um so häufiger gehen Faserzùge aus dem Tarsus in die Bindehaut über. Während hier die den Bündeln anliegenden Bindegewebszellen mit ihren deutlich hervortretenden ovalen Kernen die einzigen zelligen Elemente bilden, kommen erst in der obersten Partie der Tarsalbindehunt lymphoide Elemente hinzu, welche nun rasch an Zahl wachsen, und die Lücken zwiseben den hier mehr auseivander weichenden Bindegewebsbündeln einnehmen. Immer bleibt aber diese Struktur noch fern von der des cytogenen oder lymphdrüsenähnlichen Gewebes. Im orbitalen Theil nimmt die lymphoide Infiltration ebenso rasch wieder ab.

Es dürte nicht uninteressant sein, hier die Bemerkung anzuknüpfen, dass die Meibom'schen Drüsen hier blos die untere Halfte der Tarsuslänge oder wenig darüber einnehmen, und der von Drüsen freie Theil in seiner mittleren Partie ein auffallend lockeres Gewebe zeigt, in welchem zahlreiche zellige Elemente enthalten sind; dieselben haben theils einen runden Kern und sehr spärliches Protoplasma, theils einen ovalen, und erscheint das Protoplasma nach zwei oder mehr Richtungen in kurze Fortsătze ausgezogen. An der vorderen und hinteren Oberfläche ist das Gewebe des Tarsus aber auch in diesem drüsenfreien Abschnitte sehr dicht, laufen die Bindegewebsbündel rorzugsweise der Oberfläche parallel won oben 
nach unten, und sind spärliche spindelförmig aussehende Zellen in die engen Saftlücken eingebettet. In dem noch freien Theile des Tarsus sind die Gefässnetze, welche die Drüsenbeeren umspinnen sollen, bereits vorgebildet, und weisen gewissermaassen der wachsenden Drüse den Weg an. Die obersten Acini sind noch klein, und die Enchymzellen selbst kleiner, weniger platt, und noch nicht oder nur andeutungsweise mit Fettlournchen erfüllt. Sehr bald gewinnen sie aber ihr bekanntes charakteristisches Anssehen.

An Stellen, wo acino-tnbuläre Drüsen im Bereich des Tarsus vorkommen (Ciaccio's glandule acinose tarso-congiuntivali), finden wir dieselben auch bier schon an dem Platze, wo sie uns beim Erwachsenen begegnen, nämlich im obersten Ende des Tarsus, nur sind sie hier noch durch eine beträchtliche drüsenlose Strecke von den obersten Acinis der Meibom'schen Drüsen getrennt. Sie enthalten noch wenig Endschlăuche, und sind mit einem schönen $(0,01-0,012 \mathrm{Mm}$. hohen) Cylinderepithel ausgekleidet.

Beim neugeborenen Kind ist die Oberfläche der Bindehaut im Bereich der zwei unteren Drittel (bisweilen noch etwas mehr, bisweisen weniger) vollkommen glatt und eben, resp. es werden flach wellige Niveaudifferenzen der Tunica propria durch das Epithel ausgeglichen; dann beginnen niedrige Hügel und cylindrische Erhebungen welche gegen den convexen Rand des Tarsus an Höhe allmälig zunehmen, und jenseits desselben rasch wieder verschwinden. (Vgl. Waldeyer, 1. c., Fig. 26.) In Bezug auf den histologischen Bau des Stromas ist die Bindehaut des Neugebornen noch in nichts wesentlich verschieden von der des Embryo in den letzten Schwangeschaftsmonaten. Die lymphoide Einlagerung hat zwar an Ausdehnung etwas zugenommen, beschränkt sich aber auf den obersten mit papillenartigen Erhebungen versehenen Theil der Conjunctiva tarsi und den Anfang der Pars orbitalis. Typisch adenoides Gewebe vermissen wir anch hier noch vollständig, wie schon Stieda und Blumberg angegeben haben. Die lymphoiden Zellen v. Gräfo's Archiv für Ophtbalmologie, XXIII.4. 
nehmen eben die Lücken zwischen den hier etwas auscinanderweichenden zarten Bindogewebsbündeln ein. Bei einige Wochen alten Kindern ist bereits in dem obersten Abschnitte des Tarsaltheils alenoides Gewebe ausgebildet; die Zahl der lymphoiden Zellen hat zugenommen und es sind die Bindegewebsbündel aufgelöst in ein feinstes Netz homogener, stärker lichtbrechender Füden, welche hier wenigstens feiner sind, als in Reich's $\mathrm{Ab}$ bildung (Fig. 4) za ersehen ist. An Knotenpunkten finden sich vielfach ovale Kerne mit etwas körniger Substanz an ihren Polen; kurz wir haben an ausgepinselten Stellen vollständig das Bild eines Lymphdrüsen-Reticulums vor uns. Von einem anastomosirenden Zellennetz, von dem Waldeyer bei jüngeren Individuen spricht, konnte ich mich nirgends überzengen - es müsste denn sein, dass in ähnlicher Weise, wie $\mathbb{C}$. Klein von der. Milz nachgewiesen hat*), endotheliale Bindegewebszellen, bei der Auffaserung und Umbildung des fibrillären Bindegewebes von den Bündeln gewissermassen abgelöst, ein zart membranöses Fachwerk bildeten, und an Durchschnitten dann als sternförmige Zellen oder feine Fädchen imponirten. Zur Begründung einer solchen Vermuthung kann ich bichts positives beibringen, weil die von mir angewendeten Methoden nicht auf eine solche Untersuchung berechnet waren. So wohl an der oberen und unteren Grenze der lymphadenoiden Strecke, als auch gegen das Tarsus- und subconjunctivale Zellgewebe $\mathrm{zu}$, kann man die allmälige Umwandlung und den Uebergang der einfachen lymphoiden Einlagerung in typisch adenoides Gewebe studiren. (Es ist dies auch angedeutet in der oben citirten Fig. 4 von Reich.) An einer grösseren Zahl von Präparaten von verschieden alten Kindern überzeugt man sich leicht von der allmälig

*) Observations on the structure of the pleen. Quart. Joum. of micr. se. 1875 , pag. 363 , pI. I. 
wachsenden Ausbreitung der adenoiden Substanz, obwohl sich Zahlenangaben im Verhältniss zum Alter nicht machen lassen, weil auch bei Kindern desselben Alters doch zu grosse Schwankungen vorkommen.

Bei Erwachsenen fand ich bei jener Reihe von Lidern, bei welcher der Papillarkörper die relativ geringste Entwickelung zeigte, und auf das hintere Drittel der Tarsalbindehaut, oder wenig mehr beschränkt war (siehe oben pag. 5), auch das adenoide Gewebe anf die mit papillenartigen Erhebungen besetzte Region und die nächst angrenzende Partie der Pars orbitalis reducirt, and darüber hinaus nur mehr vereinzelte Iymphoide Zellen in Spalträume des Bindegewebes eingelagert. In der grossen Mehrzahl von Lidern, die man zur Untersuchung bekommt, ist aber die Ausdehnung des Papillarkörpers, resp. des Furchennetzes so wobl nach oben, als nach unten eine grössere, und da trifft man auch das adenoide Gewebe über die ganze hintere Hälfte der Tarsalbindehaut und einen mehr oder weniger grossen Abschnitt der Pars obitalis ausgebreitet. Anfangs nur auf die Papillen beschränkt, betrifft es dann die Schleimhaut in ihrer ganzen Dicke, und verliert sich gegen den Fornix zu allmälig wieder, indern es zuletzt nur mehr auf die oberfiächlichste Schicht der Mucosa reducirt ist, un weiterhin einer lockeren Durchsetzung des Gewebes mit vereinzelten Lymphzellen zu weichen. In jenen Fällen, welche wir oben in Bezug auf die Entwickelung des Papillarkörpers an die äusserste Grenzo des physiologischen verweisen mussten - so weit sich hier überhaupt Grenzen ziehen lassen - finden wir, bei entsprechender Zunahme der Mächtigkeit der Tunica propria conjunctivae im Ganzen, auch die adenoide Beschaffenheit des Gewebes mehr oder weniger nahe gegen den freien Lidrand sich erstreckend und nach rückwärts bis in die Uebergangsfalte reichend. Lider, 
wie sie mir anch zuweilen vorkamen, bei welchen die adenoide Substanz schon unmittelbar uber der inneren Lidkante begann, müssen entschieden für pathologisch erklärt werden, was sich auch durch die Anwesenheit zahlreicher "Schleimzellen" im Epithel kund gab.

In allen diesen Fällen konnte man sich deutlich überzeugen, dass das Auftreten einer homogenen Grenzmembran, welche von Stieda, Ciaccio, Reich und Morano beschrieben wird, streng an die Ausbildung adenoiden Gewebes geknüpt ist. So lange und so weit typisch adenoides Gewebe noch fehlt, ist, an Schnitten wenigstens, von einer Begrenzungsschicht, welche sich irgendwie, etwa durch ihre Lichtbrechung, vom unterliegenden Bindegewebe unterschiede, nichts zu sehen, so wenig wie an der Conjunctiva scleroticae, der eine homogene Grenzmembran ebenfalls abgeht. An letzterer kownte ich mich allerdings überzeugen, dass die das Epithel unmittelbar tragende dünnste Bindegewebslage viel fester und dichter gewoben ist, und macerirenden Reagentien einen viel grösseren Widerstand entgegensetzt, als die darunter liegenden Strata**) Eine solche dichtere subepitheliale Lage ist nun wohl auch an der Oberfäche der Conjunctiva tarsalis und orbitalis anzunehmen; und diese ist es dann, welche, wenn das fibrilläre Bindegewebe der Tunica propria in ein Reticulum umgewandelt ist, als Grenzmembran zu-

*) Ich machte diese Beobachtung bei Gelegenheit der Unter. suchung der Ausbreitung und Endigung der Nerven in der Bindehaut, und will hier bemerken, dass ich mich in allem Wesentlichen an Cia ccio's Darstellung (pag. 34-41), und speciell in Bezug auf die Krause'schen Endkolben den Resultaten der sorgfältigen nnd eingehenden Arbeit ron Longworth und Waldeyer (Max Schultze's Arch. f. micr. Anat. XI, pag. 653-660 und Taf. XLIV) rückhaltslos anschliesse. Nur von der Existenz der fiocchetti nervosi terminali Ciaccio's (1. c, pag. 37) habe ich mich ebenso als Longworth überzengen können. 
rückbleibt, in die die Bälkchen des Reticulums auslaufen, und welche ganz ebenso wie diese homogen und stärkex lichtbrechend erscheint, und die Essigsäure und Natronlösung stark aufquillt. Ciaccio giebt noch an, dass sie nach Einwirkung von hypermangansaurem Kali sich in zarte Fäserchen auföst (l. c., pag. 8).

Wir haben gesehen, dass bei der fortschreitenden Entwickelung der Bindebaut die Conjuntiva tarsi von der letzten Zeit des intrauterinen Lebens an ein rascheres Wachsthum besitzt als ihre Unterlage, und sich somit in demjenigen Abschnitte, wo ihre Verbindung mit der letzteren weniger fest ist, $d$. i. gegen den convexen Rand des Tarsus zu, falten muss. Nur findet die Faltung, diese Vergrösserung der Oberfläche in einer ganz bestimmten and eigenartigen Weise statt, indem halbkugelige oder cylindrische Höckerchen auftreten, welche durch das bekannte Furchennetz von einander getrennt werden. (Vgl. auch Wolfring, I. c., pag. 164.) Ziemlich gleichen Schritt mit dieser Oberflächenvergrösserung bält die Ausbildung adenoiden Gewebes; ja es ist die wachsende lymphoide Einlagerung wohl mit ein Haupt. faktor der Massenzunahme der Bindehaut. Wie haben ferner gesehen, dass in diesen beiden Beziehungen grosse individuelle Schwankungen vorkommen. Es ist schwer zu entscheiden, ob dieselben einfach in dem strafferen oder weniger straffen Anliegen der Lider an den Bulbus, also einer mehr oberflächlichen oder tieferen Lage des letzteren, resp. einer geringeren oder grösseren Länge der Lidspalte - zum Theil wenigstens - ibren Grund baben; oder ob der Zustand, wo die Erhabenheiten und die adenoide Einlagerung über die Mitte des Tarsus hinaus gegen den Lidrand hin ausgebreitet erscheinen, nicht schon als pathologisch, als das Resultat eines Reizzustandes aufzufassen sei, wie er an den Bindehäuten der Menschenklasse, deren Lider wir hauptsächlich zur ana- 
tomischen Untersuchung bekommen, in Leben häutig angetroffen wird. Ss verwischt sich eben hier in der That die Grenze zwischen dem physiologisehen und pathologischen Zustande vollständig. Ein Umstand ist meines Erachtens noch besonderer Beriicksichtigung werth, d. i. dass auch die unter dem Einflusse pathologischer Reize auftretende Schwellung und Rundzelleninfiltration sich nicht etwa unter dem Bild einer gleichmässigen Verdickung der Tunica propria conjunctiva und einer Vergrösserung der vorhandenen Papillen ausprägt, sondern nebst diesem zum Hervortreten mehr oder weniger zahlreicher never papillenartiger Erhebungen führt, und zwar nach beiden Richtungen hin, so wohl auf den ersten Falten der Pars orbitalis, die normaliter in ihrem weitaus grössten Antheile glatt ist, als auch gegen den Lidrand $z u$, indem hier Kleinwellige, Aachhügelige Unebenheiten, welche durch das Epithel sonst vollkommen ausgeglichen werden, zu Papillen sieh crheben, die durch seichtere oder tiefere Einschnitte getrennt sind, so wie sie unter normalen Verhältnissen erst in der hinteren Hälfte des Tarsus und gegen die Winkel zu angetroffen werden; wud dass ferner die Iymphoide Infiltration in der Form des typisch adenoiden Gewobes mehr oder weniger weit gegen den freien Lidrand vorrückt. Besonders instructiv war: in dieser Beziehung die Bindehaut eines wenige Wochen alten Kindes; ${ }^{*}$ denn während, wie wir gesehen haben, im normalen Zustande Papillen nur im hintersten Drittel dex Tarsalbindebaut vorkommen, und adenoides Gewebe noch gar nicht vorhanden, oder in den ersten Anfängen auf die Papillenspitzen beschränkt ist, erstrecken sich hier papillenartige Erhebungen sowohl auf den Anfang

*) Es handelto sich um einen mässigen katarhalischen Reizzustand dex Bindehant: 
der Pars orbitalis als auch etwas über die Mitte des Tarsus nach abwärts, und nahm ein typisch adenoides Gewebe die cben genannte Region so wie den übrigen Theil der Pars orbitalis bis zur Uebergangsfalte ein; auch eine Basalmembran fehlte nicht im Bereich der adenoiden Strecke. (Diess gilt von der mittleren Partie des oberen Lides; gegen die Winkel zu rückte die Entwickelung von $\mathrm{Pa}$ pillen und adenoiden Gewebe noeh näher an den freien Lidrand hin; am unteren Lide waren die Veränderungen der Tarsalbindehaut weniger ausgesprochen, hingegen die Uebergangsfalte stärker geschwellt. Die Berück* sichtigung dieser eben angeführten Verhältnisse giebt wohl eine weitere Stütze für die oben aus den morphologischen Eigenschaften der normalen Bindehaut (Gleichartigkeit der Capillaranordnung und des Epithels) abgeleitete Ansicht, dass zwischen den Papillen und den Einsenkungen keinerlei etwa auf die Function oder Bedentung dieser Gebilde sich beziehender Unterschied besteht, sondern, dass es sich bei dieser anatomischen Einrichtung lediglich um eine Oberflächenvergrösserung handle. Ebenso sieht man in Bezug auf das adenoide Gewebe, dass demselben wohl keine besonders hohe Wichtigkeit für die Function der Conjnnctiva tarsi und orbitalis beigemessen werden kann. Denn wenngleich es, mit Ausnahme der Bindehaut des Neugeborenen, nie ganz vermisst wird, so ist doch seine Ausdebnung in manchen Fällen, wie wir gesehen haben, eine recht geringe, und überhanpt in hohem Grade schwankend; es führt eben jede reichlichere Anhäufung lymphoider Zellen in der Conjunctiva tarsi und orbitalis (so lange sie eine gewisse Grenze nicht überschreitet) zur Herstellung adenoiden Gewebes; und so sehr auch das letztere nicht nur in der Anordnung seiner Elemente sondern auch in dem optischen Verhalten seiner Fasern vom fibrillären Bindegewebe sich unterscheide, so finden 
wir doch sehon im normalen Zustand der Bindebant zahlreiche Uebergänge der beilen Bindegewebsarten, und grsehen aus den angedeuteten pathologischen Vorgängen, dass nicht nur das erstere aus dem letzteren entstehen kann, sondern auch, da diese Vorgänge - soweit wir lies wenigsteñ klinisch constatiren können -- eine vollständige Restitutio ad integrum zulassen, adenoides Gewebe sich mit dem allmäligen Schwinden der lymphoiden Zellencinlagerung in gewöhnliches fibrilläres Bindegewebe zurückzubilden vermag. Es feblt auch nicht an analogen Vorgängen an anderen Localitäten des Körpers, auf die jeh bier nicht näher eingehen kann.

Was nun noch die Frage nach dem Vorkommen der Lymphollikel in der Bindehant des Menschen anlangt, so kann ich nur mit Waldeyer and Reich versichern, dass ich bei der grossen Zahl won untersuchten Liden niemals, an keiner Localität der gesunden Conjunctiva Lymphfollikel angetroffen habe, und das Vorkommen solcher Gebilde beim Menschen als entschieden pathologisch zu betrachten ist. Ciaccio, der sie unconstant und in geringer Zahl bein Mensehen gefunden zu haben angiebt, beschreibt sie als nicht deatlich abgegrenzte, nur durch eine dichtere Anhäufung der lymphoiden Zellen ron der umgebenden reticulirten Bindesubstanz sich unterscheidende Bildungen, und fugt noch hinzu, dass in den meisten der Fälle, wo sie sich vorfanden, die Schleimhaut aussergewöhnlich stark mit lymphoiden Zellen infiltrirt und mit Papillen von verschiedener Gestalt und Grösse besetzt war. (l. c., pag. 27 und 28).

Bekanntlich kamen auch Stromeyer, Blumberg und Wolfring zur Ueberzeugung, dass die sogenannten Trachomdrüsen oder Trachomfollikel in's Gebiet des Pathologischen gehörten, gingen aber wieder entschicden $z$ weit, wenn sie die Trmphfollikel bei denjenigen 
Thieren, bei denen sie im erwachsenen Zustande nie vermisst werden, ebenfalls als nicht normal functionirende Gebilde, als krankhafte Producte betrachten. Wenn auch bei den Hausthieren die Lymphfollikel der Conjunctiva in Anzahl und Ausbildung nicht immer constant gefunden werden, so ist doch, wie die Schmid'schen Untersuchungen*), die ich im vollen Maasse bestätigen kann, zuerst bestimmt ergeben haben, ihr Vorkommen ein so beständiges, ibr Fundort für die verschiedenen Specics so charakteristisch und consequent, endlich die Art und Zeitdauer ihrer Entwickelung eine so gesetzmässige, dass wir von allen sonst leitenden Gesichtspunkten bei der Beurtheilung des Normalen und Pathologischen abweichen müssten, wollten wir diese Gebilde als etwas krankhaftes ansprechen. Sehr beachtenswerth ist auch die Bemerkung, die Schmid hinwirft (l. c., pag. 13), dass wir in der Bindehaut der Thiere niemals die weiteren Folgezustände antreffen, die sich beim Menschen an die Production jener analogen Gebilde anschliessen. Das Unrichtige liegt eben darin, dass man das bei Thieren Gefundene auf Grund einzelner anologer Beobachtungen beim Menschen ohne weiteres auf diesen iibertragen hat. Schon im Entwickelungsgang der Conjunctiva liegt ein beachtenswerther Unterschied. Während bei den neugeborenen Thieren das Stroma der Bindehaut in den Regionen, wo wir später Lymphfollikel finden, ein diffus adenoides ist, weiches in dem Maasse, als die Ausbildung der Follikel vorschreitet, mehr und mehr schwindet, um einem zellenarmen fibrillären Bindegewebe Platz zu machen, so seben wir beim neugeborenen Kind bloss an einer ganz umschriebenen Stelle in der Gegend des convexen Randes des Tarsus eine lockere diffus Iymphoide Infiltration, aus welcher

*) Lymphfolliknl de: Bindehant des Auges, Wịn 1871. 
sich nachher typisch adenoides Gewebe entwickelt. Ditses letztere persistirt aber dano auch als solches in einem mehr oder weniger grossem Antheil der Conjunctiva. tarsi und orbitalis für Lebenszeit.

Es seien mir zum Schlusse noch ein Paar Bemerkungen erlaubt bezüglich der von Waldeyer sehr passend sog. acinotubulären Drüsen der Conunctiva. Was ihren feineren Bau betrifft, so hat Waldeger in kurzen Zägen die von allen Beschroibungen zutreffendste gegeben (1. c., pag. 238), und ist derselben nichts hinzuzufügen. Nur bezüglich des Sitzes dieser Drüsen herrschen noch einige Differenzen.

Ueber die im Uebergangstheil gelegenen Dräschen, welche unter dem Namen der Krause'schen Drüsen (glandes sous-conjonctivales, Sappey) bekannt sind, ist man einig, dass sie in Anzahl, Grösse und Lagerung sehr varïren können. Im Durchschnitt findet man wohl etwa 10 bis 16 in der obern (Kranse hat einmal 42 gezählt), und 2 bis 6 in der unteren Uebergangsfalte. Die obere enthält Drüschen ihrer ganzen Ausdehnung entlang, jedoch mit wachsender Zahl und Grösse gegen den äusseren Winkel zu. Am häufigsten sind sie an der Umschlagsfalte selbst anzutreffen; gegen den Rand des Tarsus hin werden sie immer sparsamer. W. Krause sah sie auch noch in einigen wenigen Exemplaren (1-4) dicht am Tarsus*), was ich vollkommen bestätigen kann. Ich fand einige Male an einem und demselben Sehnitte (ziemlich aus der Mitte des Lides) eine ganze Kette von Drüschen, von der Uebergangsfalte bis an den convexen Rand des Tarsus reichend, und selbst noch ein Weniges über denselben herab an dessen Vorderseite, wo sie dann wie in einer kleinen Nische dem Tarsus angeschmiegt lagen. An anderen Schnitten aus der-

*) Zeitschrift f. rationelle Medizin. 1854. pag. 337. 
selben Region fanden sich dann auch solche Drüschen im Gewebe des Tarsus selbst gelegen, so dass also stellenweise wenigstens ein continuirlicher Uebergang zwischen beiden Fundorten vermittelt wird. Was nun diese im oberen dreikantig-prismatischen Ende des Tarsus eingebetteten Drüsen betrifft;), welche Ciaccio, Sappey's Nomenclatur folgend, ganz passend Glandule tarso-congiuntivali nennt, so sind die Angaben über ihren Fundort zum Theil geradezu einander entgegengesetzt. Während Klei in darüber gar nichts aussagt**), versetzen sie Wolfring und Waldeyer an die nasale Hälfte des Lides***) und Ciaccio bezeichnet dies wieder als einen "grossen Irrthum", und behauptet, dass sie constant in der Gegend des äusseren Winkels zu finden sind $\dagger$ ).

Ich selbst fand sie wiederholt in schön entwickelten Exemplaren an Schnitten, die aus der Mitte des Lides stammten, und von da weg gegen die innere Halfte hin. Da nun kein Grund vorliegt, an der Richtigkeit der verschiedenen Angaben zu zweifeln, so geht daraus nur hervor, dass sich überhaupt kein für alle Fälle gültiger Standort für diese Drüschen angeben lässt, sondern, dass

*) Dass W. Krause diese Drüsen auch schon gekannt bätte, wie Reich vermuthet (l. c., pag* 17 Anm.), muss als unrichtig bezeichnet werden. Es geht dies mit Bestimmtheit hervor aus einer Stelle in W. Krause's Abhandlung über die Anatomie der Conjunctiva in L. de Wecker's Traité thér. et prat. des mal. des yeux (deuxième Edit., pag. 8), wo er sagt: Je n'ai jamais rencontré des glandes ni dans la portion palpébrale, ni dans le portion bulbaire de la conjonctive.

**) Stricker's Handbuch der Lehre von den Geweben. 1872. pag. 1148.

米落) W olfring Centralblatt f. d. med. Wissensch. 1872. No. 54, und Beitrag zur Lehre von den contagiösen Augenentzündungen. Beilageneft zu d. klin. Monatsbl. für Augenheilk. 1874. Waldeyer, 1. c., pag. 238.

†) 1. c., pag. 20 . 
sie ebenso wie an Zahl (Wolfring, Beitrag z. Lehre v. d. cont. Augenentz. pag. 12), anch in ihrem Sitz in hohem Grade variiren können.

In Bezug auf ihren feiveren Bau möchte ich nur bemerken, dass ich weder beim Nengeborenen noch beim Erwachsenen finden konnte, dass die Drüsen von reticulirter Bindesubstanz umschlossen würden, welche aus verzweigten Zellen mit in den Netzmaschen eingelagerten Lymphkörperchen bestände, wie $\mathrm{C}$ iaccio angiebt, and worauf er grosses Gewicht legt. (1. c., pag. 21). Icli fand immer nur, dass die Drüsen unmittelbar in's Gewebe des Tarsus eingebettet sind, dessen weit auseinander weichende Faserbundel sie aufnehmen, und auch die zwischen die kleineren Abtheilungen der Drüsen sich einsenkenden Septa abgeben. Auch die feineren Verzweigungen des Stromas, welche als Träger der zahlreichen Gefässe den Schlauchenden überall hin folgen, bestehen aus gewöhnlichem fibrillärem Bindegewebe, welches bloss hier lockerer und zarter ist. Im normalen Zustande finden sich nur ganz vereinzelte lymphoide Zellen darin, ebenso wie im Bindegewebe dor K rausc'schen Drüsen. Dieselben sah ich aber schon bei mässigen entzündlichen Reizungen der Bindehat an Zahl sehr beträchtich vermehrt (Vgl. Wolfring, Beitrag zur Lehre etc., pag. 21 ff.), onne dass das umgebende Gewebe (subconjunct. Bindtgewebe, resp. Tarsus) gleichzeitig Sitz zelliger Infiltration wäre. Solchen nicht mehr normalen Präparaten ist offenbar Ciaccio's Beschreibung entnommen (wir haben ja dasselbe bei seiner Darstellung der Histologic der Conjunctiva gesehen); und ausserdem ist wohl sein Netz sternförmiger Zellen (Fig.22) Product der Goldbehandlung. 